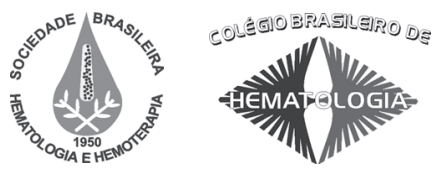

\title{
Perfil do doador de sangue autoexcluído no Hemocentro Regional de Uberaba-MG (HRU) no período de 1996 a 2006
}

\author{
Self-exclusion profiles of blood donors of the Regional Blood Bank in Uberaba, Brazil (HRU) in \\ the period of 1996 to 2006
}

\author{
Paulo R. J. Martins ${ }^{1}$ \\ Raquel A. Martins ${ }^{2}$ \\ Hélio Moraes-Souza ${ }^{3}$ \\ Valdirene F. Barbosa ${ }^{4}$ \\ Gilberto A. Pereira \\ José M. J. Eustáquio ${ }^{6}$ \\ Guilherme M. Lima ${ }^{6}$
}

\begin{abstract}
Candidatos a doação são submetidos a triagem clínica e sorológica para minimizar o risco de transmissão de doenças via transfusão. Uma de suas limitações é a janela imunológica, que possibilita a transfusão de sangue contaminado. O objetivo deste trabalho foi avaliar o índice de autoexclusão de acordo com idade, gênero, estado civil, cor e tipo de doação, as variações anuais de autoexcluídos e sua eficácia em evitar a transfusão de sangue contaminado. Os dados foram analisados através do teste qui-quadrado, odds ratio e regressão linear. De 1996 a 2006, o Hemocentro Regional de Uberaba (HRU) coletou 176.097 bolsas de sangue, das quais 2,72\% foram desprezadas por autoexclusão, com significativo predominio de homens, maiores de 29 anos, solteiros, não brancos e primeira doação $(p<0,0001)$. Observouse associação entre fidelização e autoexclusão, sugerindo que maior fidelização contribui para menor autoexclusão. A sorologia positiva para HIV1 (0,35\%) e HIV2 $(0,23 \%)$ foi significativamente maior nos autoexcluidos $(p<0,0001)$, significância não observada para $\mathrm{HCV}(0,52 \%)(p=0,24)$. Nos não autoexcluidos, estes percentuais foram de 0,15\%,0,03\% e 0,41\%, respectivamente. A maior frequência de autoexclusão em homens maiores de 29 anos, solteiros e não brancos está de acordo, em parte, com o perfil do doador do HRU. O decréscimo de 1996 a 2001 é explicado por fatores comportamentais como criação do Centro de Testagem Anônima e maior fidelização dos doadores ao longo dos anos. A maior frequência de positividade nos autoexcluídos e três soroconversões em doações subsequentes reforçam a importância dessa ferramenta na diminuição do risco de janela imunológica. Rev. Bras. Hematol. Hemoter. 2009;31(4):222-227.
\end{abstract}

Palavras-chaves: Doação de sangue; autoexclusão; sorologia positiva; segurança transfusional.

\footnotetext{
${ }^{I}$ Coordenador do Hemocentro Regional de Uberaba/Fundação Hemominas. Professor Adjunto IV de Hematologia e Hemoterapia Universidade Federal do Triângulo Mineiro - Uberaba-MG.

${ }^{2}$ Aluno do Curso de Medicina da Universidade de Uberaba - Uniube - Uberaba-MG.

${ }^{3}$ Professor Titular de Hematologia e Hemoterapia da Universidade Federal do Triângulo Mineiro. Chefe da Disciplina de Hematologia e

Hemoterapia - Uberaba-MG.

${ }^{4}$ Professora da disciplina de Bioestatística da Uniube - Uberaba-MG.

${ }^{5}$ Professor da disciplina de Bioestatística da UFTM - Uberaba-MG.

${ }^{6}$ Aluno do Curso de Medicina da Universidade Federal do Triângulo Mineiro (UFTM) - Uberaba-MG.
}

Trabalho realizado no Hemocentro Regional de Uberaba $(M G) /$ Fundação Hemominas - Uberaba-MG.

Correspondência: Paulo Roberto Juliano Martins

Av. Getulio Guarita $n^{\circ} 250-$ Abadia

38025-440 - Uberaba-MG - Brasil

Tel.: (55 34) 3312-5077

E-mail: hemocentro@mednet.com.br

Doi:10.1590/S1516-84842009005000054 


\section{Introdução}

O candidato a doação de sangue deve ser submetido a triagem clínica, hematológica e sorológica com o objetivo de proteger o doador e minimizar o risco de transmissão de doenças via transfusão sanguínea, conforme preceitua a legislação brasileira. Uma vez que a triagem clínica se baseia na resposta dos doadores a perguntas predeterminadas, estes podem omitir fatos que os colocariam no grupo de risco para certas doenças passíveis de transmissão pelo sangue. Assim, a limitação destes procedimentos reside na confiabilidade das informações prestadas pelo doador à triagem clínica, somada à possibilidade de "janela imunológica" na triagem sorológica, o que possibilita a liberação de sangue infectado para transfusão. .,2,3,4 $^{2}$

A autoexclusão foi implementada nos Estados Unidos, por recomendação da FDA (Food and Drug Administration) em 1983, com o objetivo de aumentar a segurança transfusional. ${ }^{1}$ Deste então, este procedimento tem sido aprimorado e expandido para vários países do mundo, como importante ferramenta para evitar liberação de sangue de doadores que se encontrem na janela imunológica para infecções virais, especialmente o HIV (Vírus da Imunodeficiência Adquirida) e a hepatite C. Os procedimentos da autoexclusão, geralmente anônimos, variam em forma e conteúdo de um serviço para outro. No Hemocentro Regional de Uberaba (HRU) foi implantado em 1996, através de uma cédula elaborada com linguagem simples e ilustrações, na qual o doador define entre duas alternativas, se faz parte ou não de grupo de risco para doenças hemotransmissíveis. O procedimento é feito em sala reservada e seu "voto" é registrado na cédula identificada apenas pelo número de registro no HRU. As cédulas com o voto SIM em alguma das questões que identificam comportamento de risco, voto duplo (SIM e NÃO) ou em branco sugerem que o sangue do doador possa não ser seguro para transfusão. Seu número de registro é então encaminhado para o descarte da respectiva bolsa, sem a identificação nominal do doador, garantindo assim o sigilo do procedimento e sua privacidade. Este doador não será excluído definitivamente e em doações posteriores (caso ele não se autoexclua novamente) seu sangue poderá ser utilizado. Entretanto, se o doador se autoexcluir em duas doações consecutivas, este será banido definitivamente do banco de doadores. ${ }^{3-5}$

Diversos estudos analisaram a aplicabilidade e eficácia da autoexclusão. ${ }^{3,6,7,8,10,14,15}$ As principais falhas identificadas estão relacionadas às dificuldades de entendimento das questões formuladas. Estudos avaliando tal questão demonstraram redução de autoexclusão de $0,70 \%$ para $0,26 \%$ quando o doador foi prévia e adequadamente orientado. ${ }^{6}$ Outro evidenciou que a autoexclusão pode aumentar em até 21 vezes a chance de se identificarem indivíduos HIV positivos. Contudo, a baixa frequência de doadores em janela imunológica e a discreta difusão do método minimizam a sua eficácia. ${ }^{4}$
Pesquisa desenvolvida no Canadá demonstrou que doadores que se autoexcluíram apresentaram índice de soropositividade para HIV significativamente maior do que os não autoexcluídos. ${ }^{7}$ Resultados similares foram encontrados em outro estudo em relação a sífilis, hepatites B e C, porém, quanto ao HTLV, o perfil sorológico foi similar aos que não se autoexcluíram. ${ }^{4}$ Pesquisa realizada nos Estados Unidos concluiu que, apesar do índice de soropositividade para HIV, sífilis, hepatite B e C ser significativamente maior no grupo de autoexcluídos, o procedimento foi pouco eficaz para impedir transmissão de doenças via transfusão no período de janela imunológica. ${ }^{15}$

A carência de estudos no Brasil sobre a autoexclusão e a conveniência de divulgação de uma experiência de mais de uma década do HRU com este procedimento motivaram a realização da presente pesquisa. Portanto, este trabalho tem como objetivos analisar o índice de autoexclusão de acordo com a faixa etária, gênero, estado civil, cor e tipo de doação, as variações anuais de autoexcluídos e sua eficácia em evitar a transfusão de sangue contaminado.

\section{Casuística e Método}

Trata-se de um estudo retrospectivo, de caráter descritivo, para o qual utilizou-se o banco de dados informatizado da Fundação Hemominas, referente aos prontuários dos doadores do Hemocentro Regional de Uberaba-HRU, no período de 1996 a 2006. Foram constituídos dois grupos: autoexcluídos (se autoexcluíram após a doação) e não autoexcluídos. Para cada um destes grupos foram apurados os dados absolutos e percentuais segundo algumas características socioepidemiológicas: o gênero (masculino, feminino), a faixa etária (menores que 29 anos, maiores de 29 anos), a cor da pele (branca, não branca), o estado civil (casado, solteiro e outros), o tipo de doação (primeira doação e doação de retorno) e a positividade da sorologia para HIV e HCV.

Os dados foram submetidos inicialmente a uma análise das frequências absolutas e percentuais e organizados em gráficos e tabelas. Para estudar associação entre as características de interesse foi utilizado o teste qui-quadrado e calculado o Odds ratio (Razão de chance). A tendência da autoexclusão e da fidelização foi verificada a partir do ajuste de um modelo de regressão linear nos períodos de 1996 a 2001 e 2002 a 2006. O nível de significância para todos os testes foi considerado de $5 \%$.

\section{Resultados}

No período de 1996 a 2006, o HRU coletou 176.097 bolsas de sangue, das quais 4.776 (2,72\%) foram descartadas devido à autoexclusão. Verificou-se que o percentual de autoexclusão foi significativamente maior para o sexo masculino em relação ao feminino $(3,0 \%$ e $1,9 \%$ respectivamente, $\mathrm{p}<0,0001)$, sendo que a chance de autoexclusão no grupo masculino 
mostrou-se 1,62 vezes maior que no grupo feminino; a faixa etária acima de 29 anos em relação à menor, 29 (2,8\% e 2,7\% respectivamente, $\mathrm{p}<0,0001)$ e a chance de autoexclusão no grupo acima de 29 anos é 1,20 vezes maior que no abaixo de 29 anos; a condição de solteiro em relação à de casado (3,1\% e $2,4 \%$ respectivamente, $p<0,0001$ ), sendo que a chance de autoexclusão no grupo de solteiro mostrou-se 1,30 vezes maior que no grupo de casado; o grupo não branco em relação ao branco $(3,1 \%$ e $2,5 \%$ respectivamente, $\mathrm{p}<0,0001)$ com chance de autoexclusão no grupo de pele não branca 1,24 vezes maior que no de branca e o grupo de doadores novos em relação ao de retorno $(3,1 \%$ e $2,6 \%$ respectivamente, $\mathrm{p}<0,0001$ ), sendo que a chance de autoexclusão no grupo de doadores novos mostrou-se 1,20 vezes maior que no de retorno. (Tabela 1)
Quanto à variação temporal do índice de autoexclusão ao longo dos 11 anos analisados, observa-se prevalência média de 2,72\% $\pm 0,84 \%$, com maior e menor pico em 1996 $(5,8 \%)$ e $2001(1,8 \%)$, respectivamente. Observa-se que no período de 1996 a 2001 houve uma forte e significativa tendência decrescente do índice de autoexclusão $(\mathrm{p}=0.002)$; o período seguinte, 2002 a 2006, apresentou índices próximos do $2,5 \%$, sem tendência significativa de mudança ( $\mathrm{p}=0.85)$. (Figura 1)

Quanto ao percentual de fidelização, verificamos comportamento inverso; no período de 1996 a 2001, houve uma forte tendência crescente, de $65 \%$ a $81 \%$ ( $y=-6434.7$ $+3.26 x ; \mathrm{r}=0.96 ; \mathrm{R}^{2}=0.92$ ); contudo, no período de 2002 a 2006 , esta tendência foi decrescente, de $83 \%$ a $80 \%$ ( $y=1684.8$ $0.8 \mathrm{x} \mathrm{r}=-0.94 ; \mathrm{R}^{2}=0.89$ ). (Figura 2 )

Tabela 1. Distribuição das doações autoexcluídas e não autoexcluídas quanto ao gênero, faixa etária, estado civil, cor e tipo de doação no HRU, no período de 1996 a 2006

\begin{tabular}{|c|c|c|c|c|c|c|c|c|c|c|}
\hline \multirow[t]{2}{*}{$\begin{array}{l}\text { Características } \\
\text { Epidemiológicas }\end{array}$} & \multicolumn{2}{|c|}{$\begin{array}{l}\text { Total de } \\
\text { doações }\end{array}$} & \multicolumn{2}{|c|}{$\begin{array}{c}\text { Total de doações } \\
\text { não } \mathrm{AE}\end{array}$} & \multicolumn{2}{|c|}{$\begin{array}{c}\text { Total de } \\
\text { doações AE }\end{array}$} & \multirow[t]{2}{*}{ OR } & \multirow[t]{2}{*}{ IC95\% } & \multirow[t]{2}{*}{$\chi^{2}$} & \multirow[t]{2}{*}{$p$} \\
\hline & $\mathrm{N}^{0}$ & $\%$ & $\mathrm{~N}^{0}$ & $\%$ & $\mathrm{~N}^{0}$ & $\%$ & & & & \\
\hline Feminino & 45.523 & 25,8 & 44.671 & 98,1 & 852 & 1,9 & - & - & - & - \\
\hline Masculino & 130.574 & 74,1 & 126.650 & 97,0 & 3.924 & 3,0 & 1,62 & $(1.51 ; 1.75)$ & 163,97 & $<0,0001^{*}$ \\
\hline 18 a 29 anos & 84.692 & 48,1 & 82.435 & 97,3 & 2.257 & 2,7 & - & - & - & - \\
\hline Acima de 29 anos & 91.405 & 51,9 & 88.886 & 97,2 & 2.519 & 2,8 & 1,20 & $(1.14 ; 1.30)$ & 139,69 & $<0,0001^{*}$ \\
\hline Casado & 83.842 & 47,6 & 81.822 & 97,6 & 2.053 & 2,4 & - & - & - & - \\
\hline Solteiro & 66.882 & 37,9 & 64.729 & 96,8 & 2.086 & 3,1 & 1,3 & $(1.20 ; 1.40)$ & 63,06 & $<0,0001^{*}$ \\
\hline Outros & 25.373 & 14,4 & 24.770 & 97,6 & 637 & 2,5 & 1,03 & $(0.94 ; 1.12)$ & 0,26 & 0,61 \\
\hline Brancos & 108.902 & 61,8 & 106.192 & 97,5 & 2.710 & 2,5 & - & - & - & - \\
\hline Não Brancos & 67.194 & 38,1 & 65.128 & 96,9 & 2.066 & 3,1 & 1,24 & $(1.20 ; 1.30)$ & 53,90 & $<0,0001^{*}$ \\
\hline Retorno & 129.282 & 73,4 & 125.980 & 97,4 & 3.302 & 2,6 & - & - & - & - \\
\hline $1^{\text {a }}$ doação & 46.815 & 26,5 & 45.341 & 96,9 & 1.474 & 3,1 & 1,20 & $(1.20 ; 1.30)$ & 45,81 & $<0,0001^{*}$ \\
\hline Total & 176.097 & - & 171.321 & - & 4.776 & - & & & & \\
\hline
\end{tabular}

Fonte: Banco de dados do Hemocentro Regional de Uberaba, $\mathrm{AE}=$ autoexcluídos, OR: Odds Ratio; IC95\%: Intervalo de 95\% de confiança para o OR; $\chi^{2}$. Teste qui-quadrado; *: Diferença significativa.

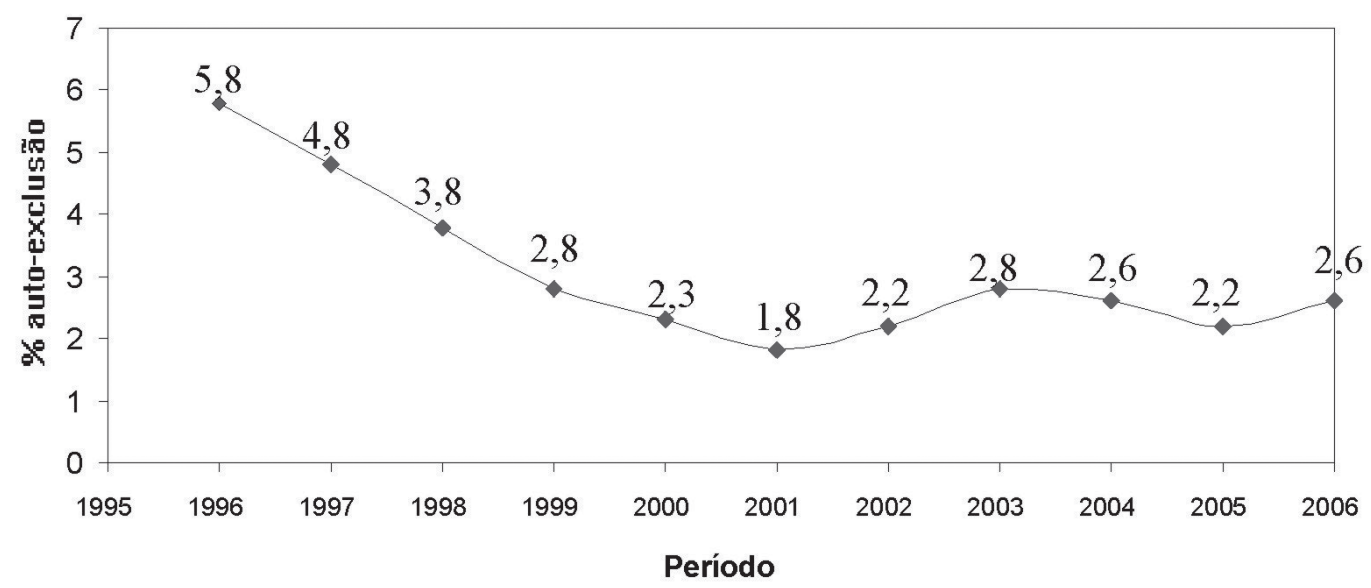

Figura 1. Percentual de doações autoexcluídas no HRU, no período de 1996 a 2006. Fonte: Banco de dados do Hemocentro Regional de Uberaba. Período de 1996 a 2001: $y=1.628-0.81 x ; r=-0.989(p=0.002) ; R^{2}=0.978$ - Período de 2002 a 2006: $y=-37.6+0.02 x ; r=0.120$ $(p=0.85) ; R^{2}=0.02$ 


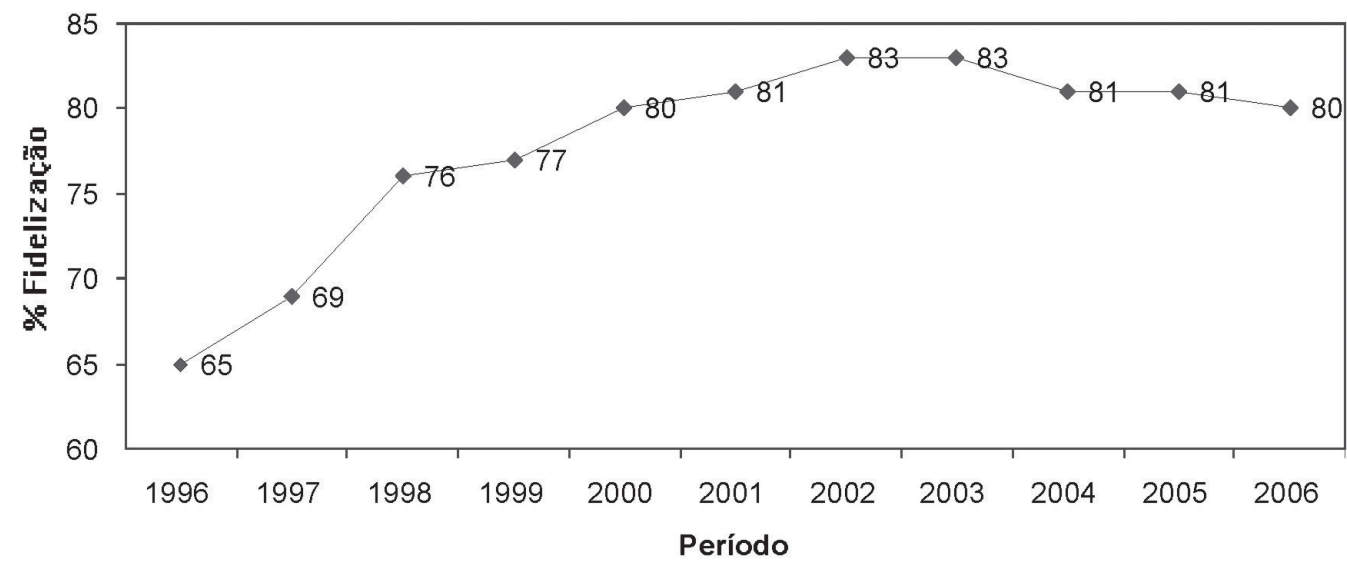

Figura 2. Percentual de fidelização das doações do HRU, no período de 1996 a 2006. Fonte: Banco de dados do Hemocentro Regional de Uberaba. Período de 1996 a 2001: $y=-6434.7+3.26 x ; r=-0.96 ; R^{2}=0.92-$ Período de 2002 a 2006: $y=1684.8-0.8 x ; r=0.94 ; R^{2}=0.89$

Tabela 2. Distribuição das doações segundo a fidelização, autoexclusão agrupadas por período no HRU, no período de 1996 a 2006

\begin{tabular}{|c|c|c|c|c|c|c|c|c|c|c|}
\hline \multirow[b]{2}{*}{ Período } & \multicolumn{2}{|c|}{ Total } & \multicolumn{2}{|c|}{ Fidelização } & \multicolumn{2}{|c|}{ Não Fidelização } & \multicolumn{4}{|c|}{ Testes } \\
\hline & $N^{0}$ & $\%$ & $N^{0}$ & $\%$ & $\mathrm{~N}^{0}$ & $\%$ & OR & IC95\% & $\chi^{2}$ & $p$ \\
\hline 1996 a 2001 & 81.183 & 46.1 & 60.562 & 74.6 & 20.621 & 25.4 & - & - & - & - \\
\hline \multirow[t]{2}{*}{2002 a 2006} & 94.914 & 53.9 & 77.450 & 81.6 & 17.464 & 18.5 & 1.51 & $(1.48 ; 1.55)$ & 1264.80 & $<0.0001^{*}$ \\
\hline & \multicolumn{2}{|c|}{ Total } & \multicolumn{2}{|c|}{ Não autoexcluídas } & \multicolumn{2}{|c|}{ Autoexcluídas } & \multicolumn{4}{|c|}{ Testes } \\
\hline Período & $N^{0}$ & $\%$ & $\mathrm{~N}^{0}$ & $\%$ & $\mathrm{~N}^{0}$ & $\%$ & OR & IC95\% & $\chi^{2}$ & $p$ \\
\hline 1996 a 2001 & 81183 & 46.1 & 78462 & 96.6 & 2721 & 3.4 & 1.57 & $(1.48 ; 1.66)$ & 233.40 & $<0.0001^{*}$ \\
\hline 2002 a 2006 & 94914 & 53.9 & 92859 & 97.8 & 2055 & 2.2 & - & - & - & - \\
\hline
\end{tabular}

Fonte: Banco de dados do Hemocentro Regional de Uberaba, AE = autoexcluídos, OR: Odds Ratio; IC95\%: Intervalo de 95\% de confiança para o OR; $\chi^{2}$ : Teste qui-quadrado; *: Diferença significativa

A Tabela 2 mostra que o percentual de fidelização no período de 2002 a 2006 foi de $81,6 \%$, valor significativamente superior ao período de 1996 a $2001(\mathrm{p}<0,0001)$, sendo que a chance de encontrar doações fidelizadas no segundo período é de 1,48 a 1,55 vezes maior que no período anterior. Já o percentual de autoexclusão mostrou-se significativamente superior no primeiro período $(\mathrm{p}<0,0001)$, com chance de autoexclusão 1,48 a 1,66 vezes maior que no segundo período. (Tabela 2)

Observamos que os índices médios de inaptidão foram maiores no grupo de autoexcluídos, sendo que para o HIV1 e HIV2 a diferença foi estatisticamente significativa, com $\mathrm{p}=0,0004$ e $\mathrm{p}<0,0001$ respectivamente; o que não ocorre para a hepatite $\mathrm{C}(\mathrm{p}=0,24)$. (Tabela 3$)$

\section{Discussão}

O perfil dos doadores de sangue autoexcluídos do HRU no período de 1996 a 2006 foi: gênero masculino, idade maior que 29 anos, solteiros, não brancos e doadores de primeira vez. Tais resultados sugerem que estes grupos (homens, idade acima de 29 anos, solteiros, não brancos e de primeira vez), devam ser alvo de maior conscientização quanto ao ato de doar e ao procedimento de autoexclusão, o que poderia contribuir para reduzir o número de bolsas descartadas e melhorar a eficácia do método. Os nossos resultados são similares ao encontrado no Canadá em 1988, em Porto Alegre em 2005 e em São José dos Campos em 2008 onde a grande maioria dos doadores autoexcluídos eram homens, solteiros, porém mais jovens. ${ }^{9,10,11}$ Estudo realizado em Araraquara em 2006 encontrou à semelhança deste estudo, maior índice de autoexclusão em doadores de maior faixa etária. ${ }^{12}$

Comparando-se aos achados da literatura, o índice de autoexclusão observado neste estudo foi bastante elevado. Assim é que, enquanto nos diversos estudos foram encontrados valores que variam entre $0,26 \%$ a $1,93 \%,{ }^{9-12}$ no HRU foram descartadas $2,72 \%$ das bolsas coletadas em consequência da autoexclusão. Esta discrepância pode ser imputada às piores condições sociointelectuais desses doadores em relação àqueles de países e/ou regiões mais desenvolvidas e, consequentemente, maior dificuldade no entendimento da ferramenta de autoexclusão. Não se deve descartar, contudo, diferenças na forma de aplicação do método de autoexclusão pelo HRU.

Observa-se ainda, no presente estudo, uma forte tendência decrescente do percentual de autoexclusão de 1996 
Tabela 3. Número e percentual de doações segundo a positividade para sorologia HIV1 e HIV2 e HCV e a condição de autoexclusão no HRU, no período de 1996 a 2006

\begin{tabular}{|c|c|c|c|c|c|c|c|c|c|c|c|c|c|c|}
\hline \multicolumn{3}{|c|}{ Doações } & \multicolumn{12}{|c|}{ Doações com Sorologia (+) } \\
\hline \multirow[b]{2}{*}{ Autoexclusão } & \multicolumn{2}{|c|}{ Total } & \multicolumn{4}{|c|}{ HIV1 } & \multicolumn{4}{|c|}{ HIV2 } & \multicolumn{4}{|c|}{$\mathrm{HCV}$} \\
\hline & $\mathrm{N}^{\circ}$ & $\%$ & $\mathrm{~N}^{0}$ & $\%$ & OR & IC95\% & $\mathrm{N}^{0}$ & $\%$ & OR & IC95\% & $\mathrm{N}^{0}$ & $\%$ & OR & IC95\% \\
\hline Não & 171321 & 97.3 & 217 & 0.75 & - & - & 45 & 0.03 & - & - & 689 & 0.41 & - & - \\
\hline \multirow[t]{3}{*}{ Sim } & 4776 & 2.7 & 17 & 0.35 & 2.47 & $(1,51 ; 4,05)$ & 9 & 0.23 & 7.19 & $(3.51 ; 14.71)$ & 25 & 0.52 & 1.30 & $(0.87 ; 1.94)$ \\
\hline & \multicolumn{2}{|c|}{$\chi^{2}$} & \multicolumn{4}{|c|}{12.54} & \multicolumn{4}{|c|}{34.75} & \multicolumn{4}{|c|}{1.41} \\
\hline & \multicolumn{2}{|c|}{ valor-p } & \multicolumn{4}{|c|}{0.0004} & \multicolumn{4}{|c|}{$<0.0001$} & \multicolumn{4}{|c|}{0.24} \\
\hline
\end{tabular}

Fonte: Banco de dados da Fundação Hemominas, OR: Odds Ratio; IC95\%: Intervalo de 95\% de confiança para o OR; $\chi^{2}$ : Teste qui-quadrado. HIV1: Elisa; HIV2: Elisa 2; HCV: Vírus da Hepatite C

a 2001, concomitante a uma forte tendência crescente do índice de fidelização. Já no período de 2002 a 2006, tanto o índice de autoexclusão quanto a fidelização se mantiveram praticamente estáveis. Portanto, parece razoável afirmar que maior índice de autoexclusão esteja relacionado a um menor índice de fidelização. Estes dados podem ainda refletir o inadequado entendimento do processo recém-implantado e a inexistência, à época, de Centro de Testagem Anônima (CTA) em Uberaba, implantado apenas em 1998. Naquela ocasião, não raramente, o setor de triagem do HRU era surpreendido pela confissão de doadores de que, com a doação, poderiam se certificar de não estarem "contaminados" (dados não publicados). Não se pode, contudo, desconsiderar a ocorrência de falhas na aplicação e, sobretudo, no entendimento do método, especialmente no seu início. Estudo realizado em Ribeirão Preto conclui que a grande maioria dos doadores que se autoexcluem não consegue captar os principais objetivos do procedimento, embora não tenham tido dificuldades de entender o instrumento utilizado. ${ }^{8}$ Pesquisa realizada em Ohio (USA), em 1990, revelou que, após campanhas explicando o que é o voto de autoexclusão e a implantação de cédulas mais didáticas e de fácil entendimento, a porcentagem de autoexcluídos passou de $0,70 \%$ para $0,26 \% .{ }^{6}$ Os autores defendem a eficácia do método, porém chamam atenção para o fato de que, além do fácil entendimento, é imprescindível que a equipe responsável pelo atendimento ao doador esteja preparada para explicar o processo e esclarecer dúvidas, diminuindo assim o descarte de bolsas de sangue e aumentando a segurança transfusional. Contrapondo-se a este, trabalho realizado em Presidente Prudente, em 2007, com o objetivo de avaliar o entendimento dos doadores quanto ao procedimento de autoexclusão concluiu que estes apresentaram um bom entendimento do processo. Entretanto, reforçam a necessidade de melhor capacitação da equipe para oferecer orientações mais adequadas. ${ }^{13}$

O presente estudo evidenciou que os doadores autoexcluídos apresentaram significativamente maior índice de soropositividade para HIV1 e HIV2, confirmando estudo realizado no Canadá em 1988. ${ }^{9}$ Para hepatite C, apesar de maior prevalência nos autoexcluídos, a diferença não foi estatisticamente significativa. Estes resultados, somados à premissa de que um paciente que se autoexcluiu possa estar temeroso de ser portador de uma doença recentemente adquirida, demonstram a utilidade do método no controle da transmissão de hepatite $\mathrm{C}$ e, sobretudo, de HIV por transfusão de sangue e, consequentemente, podem contribuir para diminuir o risco de janela imunológica.

Neste trabalho, durante a coleta dos dados, nos deparamos com três casos (já identificados pelo HRU) de doações autoexcluídas com viragem sorológica em menos de seis meses após sua autoexclusão. Destes, dois casos de hepatite C e um caso de HIV com sorologias confirmatórias (dados não mostrados).

Contudo, contrapondo-se aos nossos resultados e ao estudo canadense, ${ }^{9}$ trabalho desenvolvido na Universidade de Campinas (Unicamp), analisando amostras de sangue de autoexcluídos e não autoexcluídos, concluiu que a autoexclusão não foi eficaz para eliminar doadores de risco. ${ }^{14}$ Entretanto, o referido estudo teve a duração de oito meses, com 17.394 doações avaliadas, enquanto que no HRU foram 11 anos de avaliação, com 176.097 amostras o que, certamente, contribui para uma maior consistência dos resultados.

\section{Conclusões}

Este estudo demonstrou que doadores do gênero masculino, maiores que 29 anos, solteiros, cor de pele não branca e de primeira vez se autoexcluem mais frequentemente. Demonstrou ainda maior prevalência de HIV1, HIV2 e HCV no grupo de autoexcluídos e associação entre a fidelização e autoexclusão, sugerindo que maior fidelização pode contribuir para um menor índice de autoexclusão. Adicionalmente, o registro de três casos de sorologias reagentes para HIV e $\mathrm{HCV}$ em doações posteriores à autoexclusão, permitem concluir que o voto de autoexclusão pode ter impacto na diminuição de risco de janela imunológica. Esses dados demonstram a necessidade de conscientizar a comunidade de que, a doação é um ato de cidadania e não de avaliação do estado de saúde do doador. 


\begin{abstract}
Blood donor candidates are submitted to clinical and serological screening to minimize the risk of disease transmission. One of the limitations of this screening is the immunological window, a period that may contribute to the transfusion of contaminated blood. The aim of this study was to evaluate self-exclusion rates related to age, gender, marital status, race, type of donation, annual variations in self-exclusion and the efficacy of self-exclusion to prevent the transfusion of contaminated blood. A retrospective study was conducted and the data were analyzed using the chi-square test, odds ratio and linear regression. Of the 176,097 blood bags collected at the Uberaba Regional Blood Center (HRU) between 1996 and 2006, 2.72\% were discarded due to self-exclusion. There was a predominance of first-time, unmarried, non-white male donors over the age of 29 years old $(p<0.0001)$. An inverse association was observed between long-term commitment and self-exclusion, suggesting that the higher the return rate, the lower the incidence of self-exclusion. Positive serology for HIV1 (0.35\%) and HIV2 (0.23\%) was significantly higher among self-exclusion donors $(p<0.0001)$, a fact not observed for $\mathrm{HCV}(0.52 \%)(p=0.24)$. The percentages of these three diseases were $0.15 \%, 0.03 \%$ and $0.41 \%$, respectively among donors not initiating self-exclusion. The higher frequency of self-exclusion among unmarried, non-white male donors over the age of 29 partly agrees with the donor profile of HRU. The decline observed from 1996 to 2001 might be explained by behavioral factors, such as the creation of anonymous testing centers and an increase in the long-term commitment of donors over the years. The higher frequency of positivity among self-exclusion donors and only three seroconversions in subsequent donations support the importance of this tool to reduce the risk of contamination due to the immunological window. Rev. Bras. Hematol. Hemoter. 2009; 31(4):222-227.
\end{abstract}

Key words: Blood donation; self-exclusion; positive serology; security transfusion.

\section{Agradecimentos}

A Heloiza das Graças de Sousa, Marise Carvalho Silveira e Marli Terezinha Ferreira Lima pelo auxílio no levantamento dos dados publicados neste estudo. À Biomédica Márcia Maria Ferreira da Silva pelas importantes sugestões na análise deste trabalho. À Fapemig (Fundação de Amparo à Pesquisa do Estado de Minas Gerais) e à Fundação Hemominas, pela concessão da Bolsa de Iniciação Científica.

\section{Referências Bibliográficas}

1. D.O.U.- Diário Oficial da União; Poder Executivo. Resolução RDC número 153, de 24 de junho de 2004.

2. Silvergleid AJ. Donor screening. Clin Lab Med. 1992;12(4):669-83.

3. Chiewsilp P, Kitkornpan S, Stabunswadigan S, Iamsilp W, Suebsaeng C. Evaluation of donor self exclusion program. Southeast Asian J Trop Med Public Health. 1993;24 Suppl 1:130-2.

4. Korelitz JJ, Williams AE, Busch MP, Zuck TF, Ownby HE, Matijas $\mathrm{LJ}$, et al. Demographic characteristics and prevalence of serologic markers among donors who use the confidential unit exclusion process: the Retrovirus Epidemiology Donor Study. Transfusion. 1994;34(10):870-6.

5. Lawson-Ayayi S, Salmi LR. Infection risk and efficacy of clinical selection techniques for volunteer blood donors. Transfus Clin Biol. 1997;4(6):513-21.

6. Kean CA, Hsueh Y, Querin JJ, Keating LJ, Allensworth DD. A study of confidential unit exclusion. Transfusion. 1990;30(8):707-9.

7. Pindyck J, Waldman A, Zang E, Oleszko W, Lowy M, Bianco C. Measures to decrease the risk of acquired immunodeficiency syndrome transmission by blood transfusion. Evidence of volunteer blood donor cooperation. Transfusion. 1985;25(1):3-9.

8. Herrera P, Fernandes ATS, Ferreira O, Ubiáli EMA, Covas DT. Avaliação do entendimento do doador de sangue no Hemocentro de Ribeirão Preto quanto ao procedimento de autoexclusão. Rev. Bras. Hematol. Hemoter. 2006;28(Supl.2):279, Abstract 748 .

9. Chiavetta JA, Nusbacher J, Wall A. Donor self-exclusion patterns and human immunodeficiency virus antibody test results over a twelve-month period. Transfusion. 1989;29(1):81-3.

10. Capra MS, Souza SR, Santos AT, Correia C, Soares A, Oliveira PR, et al. Autoexclusão em doadores de sangue do Complexo Hospitalar Santa Casa de Porto Alegre: perfil comparativo com o grupo que optou pela não autoexclusão. Rev. Bras. Hematol. Hemoter. 2005;27(Supl. 2):256, Abstract 692.

11. Monteiro RFG, Kleine W, Lima PGM, Marques CM, Dorta CB, Pontes PAM, et al. Voto de autoexclusão: Características dos doadores de sangue do serviço de Hematologia e Hemoterapia de São José dos Campos. Rev. Bras. Hematol. Hemoter. 2008;30(Supl. 4):302, Abstract 783 .

12. Viola $\mathrm{CB}$, Kitamura M, Alonso BH, Machado C, Bonfá R. Avaliação do perfil dos doadores que se auto-excluíram no Hemonúcleo Regional de Araraquara de janeiro a junho de 2006. Rev. Bras. Hematol. Hemoter. 2006;28 (Supl. 2):279, Abstract 749.

13. Santos ECN, Larezzo AVEM, Norcia AMMI, Souza JF. Avaliação do entendimento do doador de sangue no Núcleo de Hemoterapia de Presidente Prudente quanto ao procedimento de autoexclusão. Rev. Bras. Hematol. Hemoter. 2007;29(Supl. 3):288, Abstract 741.

14. Landy SM, Milaré MS, Castro V. Autoexclusão: Impacto na qualidade do sangue. Rev. Bras. Hematol. Hemoter. 2004;26(Supl. 2):220, Abstract 679.

16. Zou S, Notari EP 4th, Musavi F, Dodd RY; ARCNET Study Group. Current impact of the confidential unit exclusion option. Transfusion. 2004;44(5):651-7.

Suporte Financeiro: Fapemig - Fundação de Amparo à Pesquisa do Estado de Minas Gerais.

Avaliação: Editor e dois revisores externos

Recebido: 16/08/2008

Aceito após modificações: 08/04/2009 